\title{
EFECTOS DE PROGRAMAS INTERPROFESIONALES TERAPÉUTICOS BASADOS EN EL JUEGO PARA NIÑOS CON DISCAPACIDADES DEL DESARROLLO
}

\author{
EFFECTS OF PLAY-BASED THERAPEUTIC INTERPROFESSIONAL PROGRAMS FOR \\ CHILDREN WITH DEVELOPMENTAL DISABILITIES.
}

\author{
María del Pilar Saa, ${ }^{1}$ Sheryl Rosin, ${ }^{2}$ Andreina Pavone ${ }^{3}$
}

\begin{abstract}
RESUMEN
Este trabajo es la continuación de un artículo publicado el año 2020 en la Revista Argentina de Terapia Ocupacional describiendo un programa grupal interprofesional y terapéutico basado en el juego ( (Рттв) para niños con discapacidades del desarrollo (Rosin, Saa y Pavone, 202O). El propósito de este articulo es analizar los efectos que tuvo la participación en dos PITBJ en 12 niños de 18 meses a 4 años por medio de dos estudios. El primero, de pre y post prueba, donde 7 niños fueron evaluados en sus habilidades motoras y cognitivas, así como por sus niveles de desarrollo funcional. El segundo utilizó un diseño de caso único, en el que se tomaron 5 medidas de comportamiento discretas a 5 niños en la fase inicial y en la fase de intervención. Los resultados mostraron una tendencia positiva que los pItвs aumentan los niveles de desarrollo funcional, las habilidades adaptativas, motoras y cognitivas en los niños estudiados y apoya los resultados de estudios similares publicados previamente (Imperatore, Chang, Gutiérrez, \& Gunter, 2016 y Acuña, Gutiérrez, e Imperatore, 2020), resaltando la importancia de la detección y la atención temprana utilizando programas con estas características. Mas aún, este estudio da luz a la viabilidad de implementación de los Ptibs en la población de Latinoamérica con resultados positivos.
\end{abstract}

\section{PALABRAS CLAVE}

Discapacidades del desarrollo, intervención temprana, investigación interdisciplinaria, terapia ocupacional.

\begin{abstract}
This work is the continuation of an article published in 2020 in the Argentine Journal of Occupational Therapy describing an interprofessional and therapeutic group program based on play (PIтBJ) for children with developmental disabilities (Rosin, Saa and Pavone, 202O). The purpose of this article is to analyze the effects that participation in two PIтвs had on 12 children aged 18 months to 4 years through two studies. The first, a pre and post test study, where 7 children were evaluated on their motor and cognitive skills, as well as their functional development levels. The second used a single case design, in which 5 discrete behavioral measures were taken from 5 children in the initial and in the intervention phase. The results showed a positive trend that the PIтBJs increase the levels offunctional development, adaptive, motor and cognitive skills in the children studied, and supports the results of similar studies previously published (Imperatore, Chang, Gutiérrez, \& Gunter, 2016 and Acuña,
\end{abstract}

1 OTD, OTR/L fundadora y directora de InterPlay Occupational Therapy for Children en Palm Beach, Florida. La Dra. Saa es parte del comité de Integración y Procesamiento Sensorial de AOTA; presidente del comité de discapacidades del desarrollo de FOTA. pilar@interplaytherapy. com. Teléfono: $+1(954) 296-3861$.

2 PhD, CCC-SLP, fundadora y directora de Palm Beach Speech-Language Specialists en Palm Beach Gardens, Florida. La Dra. Rosin es afiliada como profesora adjunta con las siguientes universidades: IHP at Mass General Hospital, University of Vermont, Rocky Mountain University of Health Professions, and Nova Southeastern University.

3 OT fundadora y directora de SOS therapy en cuidad de Guatemala, Guatemala 
Gutierrez, and Imperatore, 2020), highlighting the importance of early detection and intervention using programs with these characteristics. Furthermore, this study sheds light on the feasibility of implementing PTIBJs in the Latin American population with positive results.

\section{KEYWORDS}

Developmental disabilities, early intervention, interdisciplinary research, occupational therapy.

Recepcionado: 07/09/2020

Aceptado: 30/11/2020

\section{INTRODUCCIÓN}

De acuerdo con el Centro para el Control y la Prevención de Enfermedades de EE.UU (CDC), cerca del 15\% de los niños americanos sufren de problemas del desarrollo, incluyendo trastornos del espectro autista (TEA), déficit atencional e hiperactividad (TDAH), trastornos de aprendizaje (TA), discapacidad intelectual (DI), y trastornos del habla y del lenguaje (THL), entre otros (Centers for Desease Control, 2019). La mayoría de los programas existentes para el tratamiento de esta población, especialmente en la infancia temprana, usan un modelo comportamental y no de desarrollo, con poca influencia de teorías de juego y de estimulación multisensorial (Landa, 2018). Sin embargo, las mejores prácticas en los programas para la primera infancia se caracterizan por: 1) ser servicios colaborativos, 2) centrados en la familia, 3) que satisfacen las diversas necesidades y prioridades de los niños y sus familias, 4) que proporcionan oportunidades de aprendizaje basadas en los intereses del niño y con opciones de juego exploratorio, y 5) que proveen educación a los padres para apoyar el aprendizaje y el desarrollo de capacidades adaptativas funcionales y sociales de sus hijos (Dunst, Bruder, Trivette, Hamby, Raab \& McLean, 2001; Dunst, 2006; Sandall, Hemmeter, Smith, \& McLean, 2005; Zwaigenbaum, Bauman, Choueiri, Kasari, Carter, Granpeesheh, et al., 2015; Guralnick \& Bruder, 2019; Workgroup on Principles and Practices in Natural Environments, 2008). La literatura también provee principios específicos a considerar para el desarrollo de habilidades en la primera infancia, así como en la prestación de servicios. Estos principios hacen énfasis en intervenciones basadas en la relación (conexión con adultos y pares) que promuevan la atención conjunta y en intervenciones sensoriomotoras y basadas en teorías de desarrollo. La prestación de servicios considera elementos de rutina, ambientes naturales y educación a los padres (CaseSmith, 2013; Case-Smith, Frolek Clark \& Schlabach, 2013; Howe \& Wang, 2013; Kingsley \& Mailloux, 2013;
Tanner, Schmidt, Martin, \& Bassi, 202O). Estos conceptos de mejores prácticas y el desarrollo de habilidades específicas en la primera infancia son consistentes con la práctica de terapia ocupacional. Los terapeutas ocupacionales (TOs) brindan intervenciones basadas en la ocupación para aumentar la participación en todas las áreas de desarrollo (física, social-emocional, cognitiva, comunicación y autocuidado) teniendo en cuenta la motivación, el compromiso con la actividad y la interacción socioemocional del individuo. Con frecuencia, TOs proporcionan intervenciones en el entorno natural de los niños para garantizar la generalización y participación óptima en ambientes específicos (American Occupational Therapy Association, 2014). No obstante, los desafíos para implementar terapia en entornos naturales incluyen conflictos entre los programas establecidos y los mandatos locales, la inexperiencia de los terapeutas brindando consultas familiares en entornos naturales y problemas fiscales y logísticos (por ejemplo, las fuentes de pago que funcionaban para modelos basados en centros no cubren el costo de la atención basada en la familia y en la comunidad), así como escasez de personal (Hanft \& Pilkington, 2000). Tomando en cuenta esta literatura, al igual que recomendaciones de un estudio positivo donde se encontró que niños entre 18 y 36 meses que participaron en un grupo de intervención temprana basado en teorías del desarrollo e integración sensorial, aminoraron los síntomas de retrasos del desarrollo (Imperatore, Chang, Gutiérrez, y Gunter, 2016); se decidió desarrollar el Programa Interdisciplinario Terapéutico Basado en el Juego (PITBJ). Combinando principios de prácticas basadas en la evidencia (PLAY Project e Integración Sensorial), el PITBJ se centra en el juego, y tienen como fin promover el desarrollo funcional de los niños fomentando habilidades como el juego simbólico, la planificación motora, el desarrollo motriz, las interacciones sociales, el desarrollo del lenguaje, así como su desarrollo académico (Rosin, Saa y Pavone, 2020). El propósito de estos estudios piloto es evaluar la efectividad de la implementación del PITBJ 
en dos ambientes diferentes, con niños de 18 meses a 4 años, diagnosticados con trastornos del desarrollo y así evaluar la viabilidad de los programas.

\section{MéTOdos}

Debido a la falta de evaluaciones estandarizadas en la población de Guatemala para medir las funciones motoras y cognitivas de los niños, en este estudio abarcó dos investigaciones cuantitativas en las cuales se evaluaron los efectos del PITBJ durante un año. Una, con diseño cuasi experimental, usando pre prueba y post prueba en la cual se evaluaron 7 niños durante un año en niveles de desarrollo socio-emocional, motor y cognitivos. La segunda consistió en un estudio de diseño de caso único A-B donde 5 niños fueron seguidos usando marcadores comportamentales discretos y análisis visual de datos.

\section{Participantes}

Los participantes para estos estudios consistieron en una muestra de conveniencia tomada de los niños y niñas que asistían a los programas PITBJ que se llevan a cabo en Florida y Guatemala. Las edades de los niños variaban entre los 18 meses y 4 años con una media (M) de 3.05 y una desviación estándar (DS) de o.59. El 91.6\% de los participantes eran varones. Todos los participantes completaron entre 9 y 12 meses del PITBJ (M 11.58 meses, SD o.9o meses) entre los años 2018 y el 2019. Las familias en el estudio representaron el rango de condiciones socioeconómicas representativas de Florida y de la ciudad de Guatemala respectivamente. Los diagnósticos más prevalentes fueron TEA (50\%) y desordenes del desarrollo asociados con trastorno de comunicación social (50\%). Todos los participantes (100\%) mostraron alguna deficiencia de procesamiento sensorial al ser evaluados con el Perfil Sensorial 2 (Dunn, 2014). La edad y el género de los cuidadores, el idioma nativo, y el nivel de educación no se analizaron, ni fueron considerados para la inclusión o exclusión de los participantes. Los 12 participantes (7 en Florida y 5 en Guatemala) cumplieron con los siguientes criterios de inclusión: (a) fueron diagnosticados con un trastorno del desarrollo, (b) residían en el Condado de Palm Beach, FL o ciudad de Guatemala, y (c) completaron la documentación para participar en el PITBJ y en este estudio. Los participantes fueron excluidos si eran (a) menores de 18 meses de edad, (b) mayores de 4 años o (c) sin un diagnóstico específico.

\section{Reclutamiento}

Se buscó un mínimo de cinco participantes para cada estudio. Cuando se identificó que los niños cumplían con los requisitos del estudio, los cuidadores fueron contactados verbalmente y por escrito para dar consentimiento firmado de incluir a su hijo(a) en la recopilación de datos para el estudio de investigación, para su futura publicación, y para grabaciones de video y fotografía. En el documento que se proveo a los padres de los participantes se reiteró la confidencialidad de la información identificativa, y se aseguró a los padres/ cuidadores que, si no participaban en el estudio de investigación, aún podrían recibir los servicios del programa grupal. La literatura de fondo se explicó a todos los padres participantes, también su papel en la investigación y publicación, así como su derecho a suspender la participación en el estudio en cualquier momento. No se requirió la aprobación del comité de ética debido a la naturaleza privada de la investigación. Esta investigación no esta asociada con ninguna institución gubernamental ni educativa.

\section{Diseño}

La variable independiente de estos estudios es la participación de los sujetos en el PITBJ. El PITBJ combina prácticas basadas en la evidencia que se centran en el juego [Proyecto de juego (PLAY) y la Integración Sensorial (IS)] y que tienen como fin promover el desarrollo funcional general del niño. En estos programas, los educadores especiales, terapeutas ocupacionales (TO) y terapeutas de habla y lenguaje (THL), presentan oportunidades donde los niños pueden desarrollar habilidades de juego simbólico, planificación motora, uso de gestos e imitación; así como el incremento de las habilidades sociales, motrices, de lenguaje, de autoayuda, y de autorregulación, a la vez que se promueve su desarrollo académico. La meta del programa es la prestación de servicios en un entorno similar al preescolar apoyando el desarrollo de los niños para la futura participación en entornos preescolares regulares. El programa sigue un protocolo establecido con anterioridad, en el cual tiene en cuenta el nivel de desarrollo funcional de los niños, en las áreas motora, cognitiva, de lenguaje, de juego, 
de autocuidado, socioemocional y sensorial. Objetivos individuales se desarrollan para cada niño basados en la evaluación inicial y las preocupaciones de los padres. El programa presenta temas mensuales a los niños, los cuales incluyen actividades educativas consistentes con los estándares de desarrollo y aprendizaje temprano de La Florida (Florida Department of Education, 2017). Estos incluyen las áreas de desarrollo físico (motricidad gruesa y fina), social-emocional, lenguaje y literatura, razonamiento matemático, ciencias, estudios sociales y expresión creativa por medio de las artes. El progreso de los niños hacia las metas es completado cada 3 meses y la información se comparte con los padres o cuidadores. Los participantes de los PITBJ tienen dos sesiones diarias en entornos enriquecidos sensorialmente. Durante esta sesión, algunos de los elementos de proceso de fidelidad a la Integración Sensorial de Ayres (ASI) (Parham, Roley, May-Benson, Koomar, Brett-Green, Burke, \& Schaaf, 2011) como garantizar la seguridad física, presentar oportunidades sensoriales, ayudar a los niños a alcanzar y a mantener niveles de alerta óptimos, desafiar la praxis, la organización de comportamiento, el control postural, motor y bilateral, asegurar que los niños sean exitosos al realizar las actividades, y apoyar la motivación intrínseca de los niños para jugar, (Parham et al., 2011) tratan de ser mantenidos.

El entorno clínico incluyó el salón similar al salón prescolar y gimnasios sensoriomotores donde los participantes tenían la oportunidad de usar equipo suspendido, escalar, construir y usar diferentes materiales y experiencias táctiles. Estos espacios cumplen con los requisitos de fidelidad del diseño físico de Integración Sensorial de Ayres (ASI) (Parham et al., 2011). Estrategias sensoriales y principios de PLAY se utilizaron durante otras actividades del día, como actividades de mesa, merienda y tiempo en círculo (ronda).

Otra consideración importante para proporcionar el PITBJ en niños en edad preescolar es la participación de los cuidadores. Filer y Mahoney (1996) propusieron que los servicios debieran ser definidos operativamente por profesionales antes del inicio del tratamiento para que los cuidadores puedan entender qué servicios se están brindando a sus hijos. Esto requiere una planificación exhaustiva y debe ocurrir con los cuidadores para analizar las estrategias utilizadas en la intervención. PITBJ trabaja para ayudar a los padres a enfrentar los desafíos de la crianza de los niños al proporcionar ideas de juego accesibles y otros recursos. Como parte de la programación, se presenta un programa mensual de educación para padres con diferentes temas de discusión. Esta es una forma de conectarse con los padres/ cuidadores y capacitarlos sobre cómo utilizar algunas de las mismas estrategias utilizadas en los PITBJ.

Las variables dependientes para el grupo de Florida son los niveles de desarrollo funcional (NDF) y los puntajes de las subpruebas cognitiva, motora fina y motora gruesa del Bayley Scales of Infant and Toddler Development III (Bayley-III) (Bayley, 2006). Para el grupo de Guatemala son mediciones de comportamientos discretos.

\section{Materiales}

Los materiales de terapia y los juguetes terapéuticos disponibles para su uso en el entorno varían, ya que están basados en temas y cambian a menudo. El gimnasio fue modificado consistentemente de acuerdo con las experiencias sensoriales y a las habilidades motoras que se quería promover en los participantes. Para el entrenamiento a los cuidadores, se proporcionó a las familias una presentación, folletos y materiales relacionados con estrategias y/o temas con ejemplos en video. Además de la variabilidad en los materiales, también hubo variaciones en el número de personas presentes durante cada línea de base y sesión de intervención. Por ejemplo, niños adicionales, terapeutas, voluntarios, etc.

\section{Instrumentos y recolección de datos}

Se creó una ficha para recolectar los datos sociodemográficos de los participantes. Los datos evaluativos recolectados de cada niño se traspasaron a otra ficha sin ninguna información identificativa para ser analizados. Los niños asistieron al programa 3 horas al día y en diferentes frecuencias ( 2,3 o 5 veces a la semana). La frecuencia e intensidad de atención al programa fue recolectada pero no analizada. Para el estudio pre y post se usaron el Bayley-III y los Niveles de Desarrollo Funcional (NDF) para evaluar el desarrollo motor, cognitivo y socioemocional de los menores.

El Bayley-III es una prueba estandarizada que está destinada a evaluar el funcionamiento del desarrollo de bebés y niños entre 1 y 42 meses de edad. Consiste en cinco escalas: Cognitiva (COG), Lenguaje (LENG) [incluye las subpruebas de comunicación Receptiva 
(CR) y Expresiva (CE)], Motor [incluye las subpruebas de Motricidad fina (MF) y gruesa (MG)], y comportamiento socioemocional y adaptativo (cuestionario para los padres). Cada ítem de las pruebas cognitivo, lenguaje y motor, se evalúan y reciben una puntuación de o o 1. Un puntaje de 1 significa que el rendimiento del niño cumple con los criterios definidos en el manual y un puntaje de o significa que no los cumple. Según la edad del niño, los puntajes sin procesar son convertidos en puntajes escalares para cada área de rendimiento usando las tablas de conversión que se proporcionan en el manual. Las puntuaciones de las subpruebas se normalizan con una puntuación media de 10 y una desviación estándar de 3 (Bayley, 2006). La versión en inglés ha informado coeficientes de fiabilidad de: 0.91 (COG), 0.87,0.86 (MF), y 0.91 (MG) (Bayley, 2006). Esta información resalta la habilidad de la prueba Bayley III para dar resultados fiables acerca de las habilidades medidas. También hay evidencia de validez discriminativa, diferenciando entre rendimiento de niños de la muestra normativa y la de niños con condiciones específicas (Bayley, 2006). En este estudio solo las subpruebas Cognitiva, Motora Fina y Motora Gruesa se usaron para evaluar a los participantes. El Bayley-III se tomó a todos los participantes en sesiones individuales al inicio de programa y al finalizar los 9 o 12 meses de participación en el mismo. Un terapeuta ocupacional ciego a la participación de los sujetos en este estudio, entrenado y con experiencia en la toma de esta evaluación, recopiló los datos de los ítems del Bayley-III. Los puntajes brutos fueron ingresados en el Software Asistente para el Punteo del Bayley-III y los puntajes escalares de las subpruebas COG, MF y MG fueron traspasados a la ficha de datos evaluativos del estudio.
Los niveles de desarrollo funcional (NDF) (Geenspan \& Weider, 1997) se midieron observando a los participantes durante 15 minutos mientras interactuaban en el PITBJ. Cada uno de los 6 niveles de desarrollo funcional (ver Tabla 1) se dividen en sub-ítems, los cuales se registran de forma escalar en una escala de Likert, de o-4, donde cada número representa un porcentaje de habilidad demostrada por el niño de esta manera: $\mathrm{O}=$ $\mathrm{O} \%, 1=25 \%, 2=50 \%, 3=75 \%, 4=100 \%$.

Los participantes fueron observados durante el inicio (primera semana) y el final (última semana) del programa por los primeros 15 minutos de participación, a una distancia de no más de cinco pies de radio de la interacción niño. Se observó la frecuencia de ocurrencia de cada nivel de desarrollo funcional (NDF) independientemente. La frecuencia de ocurrencia de cada NDF fue calificada por un terapeuta de lenguaje ciego a la participación de los sujetos en el estudio y se traspasaron a la ficha de datos evaluativos respectivamente.

Para el estudio de caso único A-B se utilizaron mediciones de frecuencia (recuentos), duración (longitud de tiempo) o magnitud (intensidad) de observaciones de comportamientos discretos en 6 áreas. 1) minutos de atención a la tarea, 2) palabras con intento comunicativo en 30 minutos, 3 ) iniciación de interacción social durante 30 minutos, 4) alimentos aceptados (solo en algunos niños que presentaban problemas de alimentación), 5) comportamientos mal adaptativos durante el programa, y 6) control de esfínter (solo los niños que presentaban esta dificultad). Solo 5 comportamientos se midieron en cada niño dependiendo de las dificultades presentadas al iniciar el estudio. Los niños fueron

TABLA 1 NIVELES DE DESARROLLO FUNCIONAL

\begin{tabular}{|c|c|}
\hline NDF 1 & Atención compartida y autorregulación \\
\hline NDF 2 & Compromiso y relación con los demás \\
\hline NDF 3 & Comunicación de 2 vías con gestos \\
\hline NDF 4 & $\begin{array}{l}\text { Comunicación de } 2 \text { vías compleja con palabras / frases que incluyen resolución de problemas y } \\
\text { regulación del estado de ánimo }\end{array}$ \\
\hline NDF 5 & $\begin{array}{l}\text { Significados e ideas compartidos en juegos de simulación, preguntas y respuestas sobre } \\
\text { preguntas }\end{array}$ \\
\hline NDF 6 & Pensamiento emocional, sentido de lógica y razonamiento y comienzo de la teoría de la mente \\
\hline
\end{tabular}


observados cada tres meses por la investigadora de sitio durante su participación en el PITBJ de Guatemala. Se tomaron muestras para la fase basal y la fase de intervención. En la fase basal, se adquirieron 5 muestras para encontrar estabilidad en los datos. En la fase de intervención se obtuvo las mismas medidas cada 203 meses, durante 9 meses o un año de acuerdo con el tiempo de participación de los niños (4 o 5 datos).

\section{Intervención}

La fase de intervención de los dos estudios fue consistente con los métodos de los PITBJ descritos en Rosin, et al. (2O2O). Los 12 participantes asistieron 3 horas al día, durante 2-5 días por semana, dependiendo del curso de tratamiento recomendado. Los niños participaban en las rutinas prescolares con una educadora especial capacitada en el Proyecto PLAY, mientras que los terapeutas ocupacionales y del habla se integraron por una hora cada una separadamente a cada sesión. Durante estos periodos, se usaron estrategias ricas en estímulos sensoriales. Al principio del día, durante 15 minutos de juego libre, donde la educadora especial y los terapeutas de lenguaje entrenados en PLAY y en estrategias sensoriales de intervención, proporcionan oportunidades de conexión social, juego y lenguaje. La segunda sesión es una hora de gimnasio, donde la terapeuta ocupacional y la educadora especial presentan actividades sensoriales (táctiles, propioceptivas y vestibulares), promoviendo la participación en el juego, actividades cognitivas, de motricidad fina y gruesa, de socialización y conexión. Cada tres meses, evaluadores independientes para el pre y post test, y ciegos a las condiciones experimentales evaluaron a todos los participantes de los PITBJ en sus habilidades sensoriomotoras y en los NDF para así, ajustar sus intervenciones al nivel de desarrollo actual de los niños. De esta manera, cuando se planeaban las intervenciones grupales, se escogían estrategias sensoriales, métodos, principios y técnicas del proyecto PLAY basadas en las necesidades $y$ fortalezas de los menores.

\section{Análisis de Datos}

Análisis descriptivos se usaron para examinar las características de los participantes. La prueba no paramétrica de rangos y signos igualados de Wilcoxon para la comparación de las medianas de las muestras emparejadas
(Pagano y Gauvreau, 1993) se aplicó para analizar los puntajes escalares del Bayley-III y de los NDF de los 7 participantes del estudio pre y post, recolectados al inicio del programa y después de 9012 meses de participación. Se indicó una prueba no paramétrica debido a los datos ordinales y la falta de distribuciones normales de puntajes para la mayoría de los ítems. La prueba de rango con signo de Wilcoxon es útil con medidas que son continuas y de nivel de intervalo (como los son los NDF - en forma Likert), pero que tienen distribuciones de mal comportamiento. Esta prueba se considera con puntajes escalares, para aumentar su potencia, aumentando la probabilidad de detector efectos estadísticamente significativos que la prueba de pares $t$ (Cleophas \& Zwinderman, 2016).

Análisis visual (Zhan \& Ottenbacher 2001) [línea de aceleración (línea de división media), y banda de desviación estándar], así como la prueba binomial, fueron utilizados para estudiar los datos de los 5 participantes del estudio de caso único AB. Se analizaron los datos graficándolos y haciendo juicios sobre si la variable independiente afectó a la variable dependiente en función del nivel (magnitud), la dirección (línea de tendencia), la inclinación (pendiente) y la latencia, así como reducciones en la variabilidad (Ottenbacher, 1984). El criterio más importante fue si el tratamiento marcó una diferencia práctica (o clínica) en los sujetos.

La latencia, es el tiempo que tarda la variable dependiente en comenzar a cambiar después de una variación en las condiciones (en este caso la introducción del programa PITBJ). En general, si un cambio en la variable dependiente comienza poco después de la variación en las condiciones, esto sugiere que el tratamiento es responsable (Nock, Michel, \& Photos, 2007).

La prueba binomial de significancia es un tipo de prueba de probabilidad. Se utiliza para examinar la distribución de una sola variable dicótoma en el caso de muestras pequeñas. Implica probar la diferencia entre una proporción de muestra y una proporción dada (Frey, 2018).

\section{Resultados}

Los resultados del estudio pre y post, indicaron que, de 7 participantes, todos excepto por uno, mejoraron en todos los puntajes escalares del Bayley III (Tabla 2) y en sus NDF (Tabla 3). El sujeto que no mejoró en todos los 
puntajes, mantuvo su puntuación de MG estable en el pre y el post-test. Los puntajes COG, MF y NDF, ascendieron consistentemente con los otros participantes.

Los resultados de la prueba Wilcoxon de rangos y signos, indicó que todas las medianas clasificadas de los puntajes de los post- test (COG, MF, MG y NDF) fueron estadísticamente significativamente mejores que las medianas clasificadas de los puntajes de los pre- test, $\mathrm{n}=7, \mathrm{Z}=\mathrm{O}, \mathrm{p}<0.05$.

Consistentemente, en el estudio de caso único A-B, todos los participantes demostraron tendencias de cambios positivos en los comportamientos discretos analizados. Todos los comportamientos medidos cambiaron en la fase de intervención. Los resultados de las pruebas binomiales de los datos de los 3 participantes a los que se les tomaron 5 datos en la fase de intervención indican que la proporción de los puntos de observación por encima de la línea de tendencia fue más de la esperada (o.50), con un nivel de significancia de $\mathrm{p}=\mathrm{O} .03$. Los otros dos participantes tuvieron solo 4 puntos de datos debido a que solo permanecieron en el programa por 11 meses antes de ser integrados al prescolar regular. El nivel de significancia para la prueba binomial de todas las observaciones de estos sujetos fue marginalmente significativo de $\mathrm{p}=0.065$.

TABLA 2 PUNTAJES DE PRE Y POST PRUEBA DEL BAYLEY-III CON SEÑALES, VALORES ABSOLUTOS Y RANGOS PARA LA PRUEBA WILCOXON

\begin{tabular}{|c|c|c|c|c|c|c|c|c|c|c|c|}
\hline & P1 & $\mathbf{P} 2$ & P3 & P4 & P5 & P6 & P7 & Mediana & $\begin{array}{l}\text { Suma de } \\
\text { Positivos }\end{array}$ & $\begin{array}{l}\text { Suma de } \\
\text { Negativos }\end{array}$ & $\begin{array}{c}\text { Valor } \\
\text { Critico a } \\
\mathbf{a}=\mathbf{0 . 0 5}\end{array}$ \\
\hline $\begin{array}{l}\text { Cognición Pre } \\
\text { Prueba }\end{array}$ & 6 & 5 & 12 & 9 & 6 & 6 & 7 & 6 & & & \\
\hline $\begin{array}{l}\text { Cognición Post } \\
\text { Prueba }\end{array}$ & 14 & 8 & 14 & 12 & 7 & 9 & 8 & 9 & & & \\
\hline Signo & -1 & -1 & -1 & -1 & -1 & -1 & -1 & & & & \\
\hline Valor Absoluto & 8 & 3 & 2 & 3 & 1 & 3 & 1 & & & & 2 \\
\hline Rango & -7 & -5 & -3 & -5 & -1.5 & -5 & -1.5 & & 0 & -28 & \\
\hline $\begin{array}{l}\text { Motricidad Fina Pre } \\
\text { Prueba }\end{array}$ & 6 & 5 & 7 & 7 & 4 & 6 & 7 & 6 & & & \\
\hline $\begin{array}{l}\text { Motricidad Fina Post } \\
\text { Prueba }\end{array}$ & 13 & 7 & 14 & 10 & 8 & 9 & 11 & 10 & & & \\
\hline Signo & -1 & -1 & -1 & -1 & -1 & -1 & -1 & & & & \\
\hline Valor Absoluto & 7 & 2 & 7 & 3 & 4 & 3 & 4 & & & & 2 \\
\hline Rango & -6.5 & -1 & -6.5 & -2.5 & -4.5 & -2.5 & -4.5 & & 0 & -28 & \\
\hline $\begin{array}{l}\text { Motricidad Gruesa } \\
\text { Pre Prueba }\end{array}$ & 6 & 6 & 9 & 6 & 4 & 4 & 8 & 6 & & & \\
\hline $\begin{array}{l}\text { Motricidad Gruesa } \\
\text { Post Prueba }\end{array}$ & 10 & 8 & 10 & 9 & 5 & 7 & 8 & 8 & & & \\
\hline Signo & -1 & -1 & -1 & -1 & -1 & -1 & 0 & & & & \\
\hline Valor Absoluto & 4 & 2 & 1 & 3 & 1 & 3 & 0 & & & & 2 \\
\hline Rango & -7 & -4 & -2.5 & -5.5 & -2.5 & -5.5 & 0 & & 0 & -27 & \\
\hline
\end{tabular}

La Tabla 2 muestra los puntajes prey post intervención de cada uno de los sujetos en las sub-pruebas de cognición, motricidad fina y motricidad gruesa del Bayley III, con los resultados de la prueba estadística de Wilcoxon. Elaboración propia. 
TABLA 3 PUNTAJES DE PRE Y POST PRUEBA DE TODOS LOS NDF CON SEÑALES, VALORES ABSOLUTOS Y RANGOS PARA LA PRUEBA WILCOXON

\begin{tabular}{|c|c|c|c|c|c|c|c|c|c|c|c|}
\hline & P1 & P2 & P3 & P4 & P5 & P6 & P7 & Mediana & $\begin{array}{l}\text { Suma de } \\
\text { Positivos }\end{array}$ & $\begin{array}{l}\text { Suma de } \\
\text { Negativos }\end{array}$ & $\begin{array}{l}\text { Valor } \\
\text { Critico a } \\
\alpha=0.05\end{array}$ \\
\hline NDF 1 Pre Prueba & 1.61 & 1.78 & 1 & 1 & 0.05 & 1 & 0.56 & 1 & & & \\
\hline NDF 1 Post Prueba & 3.11 & 3.32 & 2.92 & 2.68 & 2.78 & 3.2 & 2.67 & 2.89 & & & \\
\hline Signo & -1 & -1 & -1 & -1 & -1 & -1 & -1 & & & & \\
\hline Valor Absoluto & 1.5 & 1.54 & 1.92 & 1.68 & 2.73 & 2.2 & 2.11 & & & & 2 \\
\hline Rango & -1 & -2 & -4 & -3 & -7 & -6 & -5 & & 0 & -28 & \\
\hline NDF 2 Pre Prueba & 2.36 & 2.34 & 1.5 & 2.21 & 0.05 & 1 & 1.67 & 1.67 & & & \\
\hline NDF 2 Post Prueba & 3.67 & 3.23 & 3.5 & 3.22 & 2.67 & 2.82 & 3.11 & 3.22 & & & \\
\hline Signo & -1 & -1 & -1 & -1 & -1 & -1 & -1 & & & & \\
\hline Valor Absoluto & 1.31 & 0.89 & 2 & 1.01 & 2.62 & 1.82 & 1.44 & & & & 2 \\
\hline Rango & -3 & -1 & -6 & -2 & -7 & -5 & -4 & & 0 & -28 & \\
\hline NDF 3/4 Pre Prueba & 2.12 & 2.27 & 2.14 & 2.27 & 1 & 1 & 1.43 & 2.12 & & & \\
\hline $\begin{array}{l}\text { NDF } 3 / 4 \text { Post } \\
\text { Prueba }\end{array}$ & 3.84 & 3.29 & 3.25 & 2.75 & 2.85 & 3 & 2.64 & 3 & & & \\
\hline Signo & -1 & -1 & -1 & -1 & -1 & -1 & -1 & & & & \\
\hline Valor Absoluto & 1.72 & 1.02 & 1.11 & 0.48 & 1.85 & 2 & 1.21 & & & & 2 \\
\hline Rango & -5 & -2 & -3 & -1 & -6 & -7 & -4 & & 0 & -28 & \\
\hline NDF 5 Pre Prueba & 0.05 & 1 & 0.01 & 2.2 & 0 & 0 & 1.07 & 0.05 & & & \\
\hline NDF 5 Post Prueba & 3.2 & 3.21 & 3 & 2.87 & 2.6 & 2.78 & 2.43 & 2.87 & & & \\
\hline Signo & -1 & -1 & -1 & -1 & -1 & -1 & -1 & & & & \\
\hline Valor Absoluto & 3.15 & 2.21 & 2.99 & 0.67 & 2.6 & 2.78 & 1.36 & & & & 2 \\
\hline Rango & -7 & -3 & -6 & -1 & -4 & -5 & -2 & & 0 & -28 & \\
\hline NDF 6 Pre Prueba & 0 & 1 & 0 & 1.23 & 0 & 0 & 1 & 0 & & & \\
\hline NDF 6 Post Prueba & 2.87 & 2.83 & 3.2 & 2.57 & 2.72 & 1.75 & 2.53 & 2.72 & & & \\
\hline Signo & -1 & -1 & -1 & -1 & -1 & -1 & -1 & & & & \\
\hline Valor Absoluto & 2.87 & 1.83 & 3.2 & 1.34 & 2.72 & 1.75 & 1.53 & & & & 2 \\
\hline Rango & -6 & -4 & -7 & -1 & -5 & -3 & -2 & & 0 & -28 & \\
\hline
\end{tabular}

La Tabla 3. muestra los puntajes pre y post intervención de cada uno de los sujetos en los niveles de desarrollo funcional 1, 2 y 3/4, con los resultados de la prueba estadística de Wilcoxon. Elaboración propia. 
FigURA 1. S4 - MINUTOS DE ATENCIÓN A LA TAREA. LÍNEA DE ACELERACIÓN, TENDENCIA, DIVISIÓN DE LA LÍNEA MEDIA

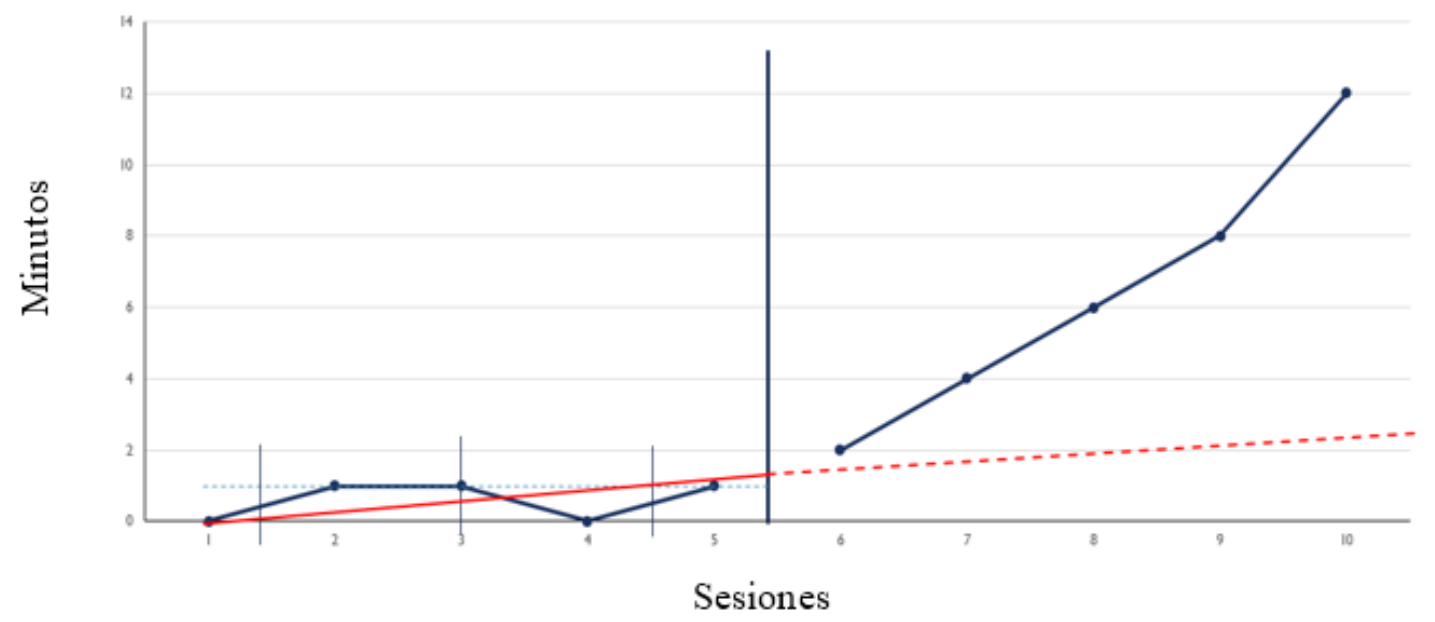

FIGURA 2. S4 - INICIACIÓN DE INTERACCIÓN SOCIAL EN 3 O MINUTOS. DESVIACIÓN ESTÁNDAR

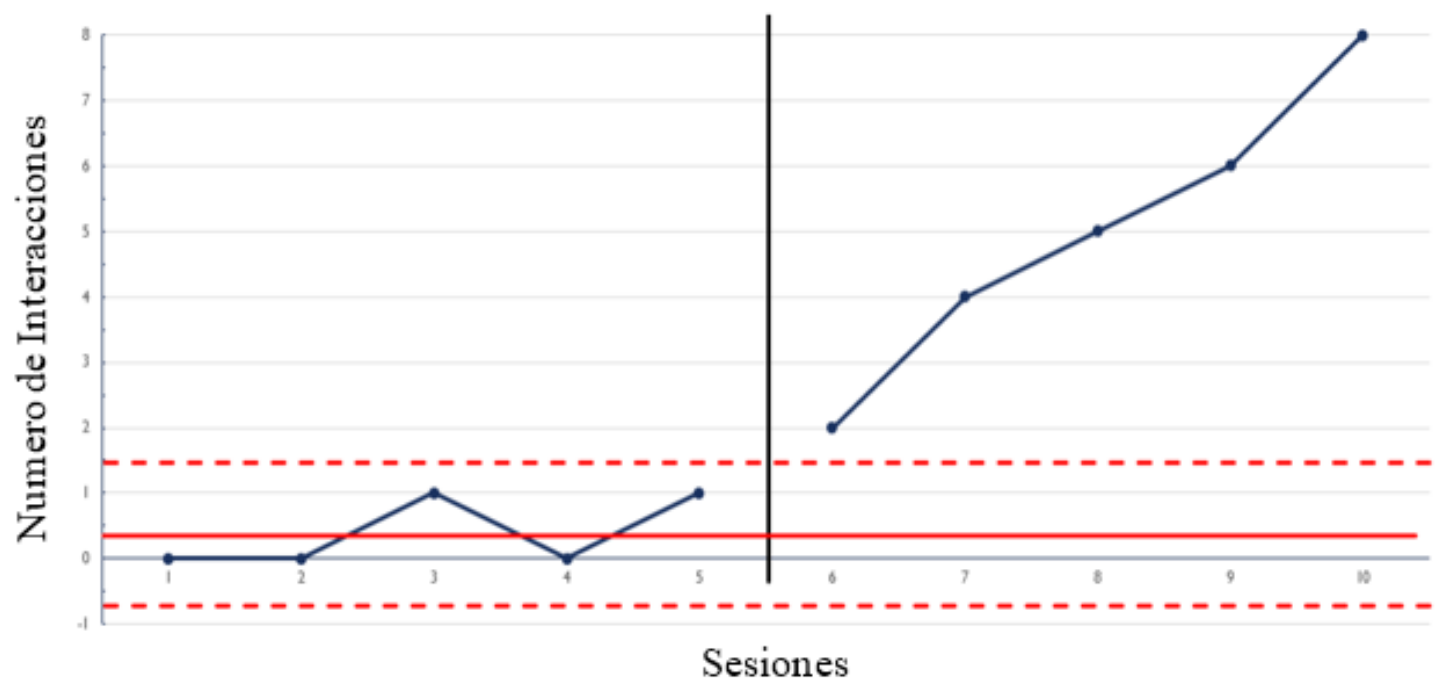

Los gráficos 1 y 2 ejemplifican el análisis visual que se realizó con todos los participantes para cada uno de los datos.

Estos resultados indican que la participación en los PITBJ, programas informados por las teorías de IS y PLAY muestran una tendencia positiva de optimización de las habilidades de desarrollo motor, cognitivo y socio-emocional de los niños que participan. Al mismo tiempo, tienen un efecto positivo durante la intervención, en el nivel de atención, comportamientos mal adaptativos, palabras con intento comunicativo, iniciación de interacción social, control de esfínteres y número de alimentos aceptados de niños y niñas entre 18 meses y 4 años. 


\section{Discusión}

Los efectos de los PITBj fueron examinados por dos estudios piloto los cuales resultaron en cambios positivos, no solo en las áreas adaptativas medidas con comportamientos discretos y en los NDF, sino también en los puntajes escalares del Bayley-III. Esto indica que las mejorías cambiaron el perfil motor y cognitivo de estos niños de estar por debajo del nivel promedio o límite con el promedio, a estar por dentro o por encima del promedio (COG y MF), comparado con el desarrollo típico de acuerdo con los resultados encontrados en el manual del Bayley-III. El área de motricidad gruesa fue la que menos mejorías reflejó, aunque todavía mostro cambios significativos en esta muestra pequeña.

Los resultados de los puntajes de los NDF indican que, aunque los niveles funcionales (NDF1 a NDF6) subieron en todos los participantes, el nivel 5 - Respuesta Socioemocional, fue el nivel que más mejoró colectivamente. En este nivel, las habilidades evaluadas incluyen capacidades socioemocionales como saludar a personas familiares, entender el significado de oraciones completas pero sencillas, demostrar sentido del humor, seguir comandos de dos pasos, comunicación con palabras o gestos en vez de acciones, habilidad de reconocer y expresar emociones de manera efectiva, participar en juegos de simulación, usar frases de una o dos palabras (no repetitivas ni estereotipadas), mostrar interés en pares, responder a preguntas, y conformarse a las normas o peticiones de los adultos o de la rutina.

En el estudio de diseño de caso único A-B, se puede concluir que hay diferencia significativa entre las fases de base y la fase de implementación del programa de intervención temprana para los 5 niños que participaron en este estudio medida por los análisis visuales y la prueba binomial. Los cambios de tendencia fueron mayores que los cambios de nivel lo que significa que la latencia fue larga y que los 5 participantes mostraron mejorías a lo largo de los 11 o 12 meses de intervención, la cual no se reflejó inmediatamente se inicio del programa.

En general, los resultados demuestran que los dos programas grupales interdisciplinarios terapéuticos basados en el juego que se estudiaron tienen tendencias a efectos positivos en disminuir los retrasos del desarrollo en las áreas motrices, cognitivas, socioemocionales y adaptativas de los niños con discapacidades en el desarrollo. Los participantes del estudio respondieron igualmente al tratamiento a pesar de tener dos tipos de diagnósticos diferentes (retrasos del desarrollo con trastornos del lenguaje y la comunicación social, y TEA - todos con dificultades de procesamiento sensorial) lo cual es un buen indicador de que el PITBJ satisface las necesidades de intervención temprana de individuos con trastornos del desarrollo que afectan el desempeño motor, sensorial, social, de lenguaje, y cognitivo indistintamente. Las tendencias positivas de este estudio reflejan los resultados del estudio a mayor escala conducido por Imperatore, Chang, Gutierrez y Gunther en 2016, replicado con una muestra significativamente mas amplia, en un estudio publicado en el 2020 por Acuna, Gutierrez e Imperatore en la revista Argentina de Terapia Ocupacional, en los cuales encontraron que un programa de intervención temprana informado por la teoría de IS y el Proyecto de Juego fue efectivo en aminorar los signos de retrasos del desarrollo en niños de 18 a 36 meses.

En el tratamiento de niños con discapacidades de desarrollo, la intervención temprana es de gran importancia, especialmente la participación en programas colaborativos, centrados en la familia, proporcionando oportunidades de aprendizaje basadas en los intereses del niño y con opciones de juego exploratorio (Dunst, 2006; Zwaigenbaum, Bauman, Choueiri, Kasari, Carter, Granpeesheh, et al., 2015; Guralnick \& Bruder, 2019). Los resultados de este trabajo proveen una dirección positiva en la implementación de programas terapéuticos aplicando estos principios con la influencia de teorías de juego y de estimulación multisensorial, y considerando elementos de rutina, ambientes naturales y de educación a los padres como lo plantean Case-Smith, 2013; Case-Smith, Frolek Clark \& Schlabach, 2013; Howe \& Wang, 2013; Kingsley \& Mailloux, 2013; Tanner, Schmidt, Martin, \& Bassi, 202O, para el beneficio del desarrollo de los menores.

\section{Limitaciones}

Una de las limitaciones de este estudio fue la diferencia de frecuencia en la que asistieron los participantes al programa. Se registraron variaciones de participación de 2 días a la semana a 5 días a la semana, pero no se realizo análisis alterno. Esto podría afectar los resultados, ya que se espera que la intensidad alta, podría optimizar el desarrollo de los niños. Otra limitación fue la 
cantidad de capacitación brindada a los cuidadores, la asistencia inconsistente a las sesiones de capacitación y la falta de registros de la frecuencia e intensidad del apoyo brindado a los niños en el hogar por los cuidadores. Esto podría interferir con los resultados, por lo que se aconseja la recopilación de estos datos en estudios futuros. Todos los niños recibieron terapia ocupacional y de lenguaje individual adicional al programa de intervención temprana añadiendo variables intervinientes a este estudio. Estas variables es necesario controlar en el futuro. Al mismo tiempo, no se puede descartar la amenaza de la historia a la validez en este estudio, debido a que los participantes no comenzaron la investigación al mismo tiempo y debido a que en el diseño de caso único A-B, el período entre la última medida de la fase de base y la primera medida de la fase de tratamiento es susceptible a efectos históricos (Salkind, 2010) donde un niño puede tener experiencias diferentes que el otro entre las pruebas previas y posteriores afectando los resultados. Por otra parte, el poder en los estudios de caso único está relacionado con el número de puntos de datos reunidos para cada participante y en este estudio se recogieron solo 4 y 5 datos limitando su poder. De la misma manera, aun cuando se encontró un nivel estable en la fase de base, la maduración es todavía una variable interviniente que se debe considerary que amenaza la validez de los estudios de caso único. La reactividad al proceso de medición puede afectar los resultados de los participantes, especialmente en niños en edades prescolares. También se requiere precaución con respecto a la generalización de los resultados actuales. La generalización de los diseños de caso único requiere replicación directa, replicación sistemática y replicación clínica ya que el análisis de datos generalmente implica la inspección visual de gráficos de las mediciones (Salkind, 2010). De la misma manera, en el estudio pre y post, la muestra fue una muestra de conveniencia pequeña y no se contó con un grupo de control.

\section{Conclusión}

Los resultados de este trabajo son relevantes y resaltan que programas de intervención temprana que siguen las recomendaciones para las mejores prácticas en los programas para la primera infancia y a su vez son combinados con teorías de estimulación multisensorial y de Proyecto PLAY, pueden ser eficaces en aminorar los síntomas de los niños con retrasos de desarrollo. Al mismo tiempo, los resultados de este trabajo respaldan estudios previos utilizando principios similares, estableciendo la importancia de evaluar el procesamiento sensorial de los niños que participan en programas de intervención temprana, para poder proveer oportunidades multisensoriales y estrategias sensoriales que suplan sus necesidades especificas.

\section{Consideraciones futuras}

Sería beneficioso replicar los estudios actuales utilizando métodos similares; pero a mayor escala y añadiendo un grupo de control para fortalecer la confiabilidad y la validez interna y externa del estudio, incrementando así el poder y la generalización de los resultados. Aunque el estudio actual sugiere que el PITBj fue efectivo, sería beneficioso investigar los efectos a largo plazo de cómo se desempeñan estos niños si se integran en los programas típicos de preescolar o jardín de infantes en el futuro. En próximas investigaciones, es importante tener más puntos de datos para controlar las amenazas potenciales a la validez interna, así como también realizar estudios comparativos de la eficacia de distintos tratamientos alternativos.

\section{Fuentes de Financiamiento}

Este estudio no estuvo financiado por ninguna institución o por ninguna donación, beca o concesión que necesite ser revelado. Los autores de este artículo no tienen relaciones financieras o no financieras relevantes para revelar.

\section{RefERENCIAS BibLIOGRÁFICAS}

Acuña, C. Y., Gutiérrez, J., Imperatore, E. (2020). Impacto del Programa Grupal Interdisciplinario de Intervención Temprana Enriquecido Sensorialmente en el Progreso de Niños con Retraso en el Desarrollo. Revista Argentina de Terapia Ocupacional, 6(1), 13-19. American Occupational Therapy Association [AOTA]. (2014). Occupational therapy practice framework: Domain and process (3rd ed.). American Journal of Occupational Therapy, 68(Suppl .1), S1- S48.

Bayley, N. (2006). Bayley-III: Bayley Scales of Infant and Toddler Development, Third ed. San Antonio, Texas, Estados Unidos: Pearson.

Case-Smith, J. (2013). Systematic review of interventions to promote social-emotional development in young children with or at risk for disability. American Journal of Occupational Therapy, 67(4) 395-404. https://doi.org/10.5014/ajot.2013.004713 
Case-Smith, J., Frolek Clark, G. J., \& Schlabach, T. L. (2013). Systematic review of interventions used in occupational therapy to promote motor performance for children ages birth-5 years. American Journal of Occupational Therapy, 67(4), 413-424. https://doi. org/10.5014/ajot.2013.005959

Centers for Disease Control. (2019). Increase in Developmental Disabilities Among Children in the United States. United States: Centers for Disease Control. Recuperado de https://www.cdc.gov/ncbddd/ developmentaldisabilities/features/increase-in-developmentaldisabilities.html

Cleophas, T.J., \& Zwinderman, A.H. (2016) Paired Continuous Data (Paired T-Test, Wilcoxon Signed Rank Test). In: Clinical Data Analysis on a Pocket Calculator. Springer, Cham. Recuperado Sep 2, 2019, de https://doi.org/10.1007/978-3-319-27104-0_6

Dunn, W. (2014). Sensory Profile 2 user's manual. Bloomington, IN: Pearson.

Dunst, C. J. (2006). Parent-mediated everyday child learning opportunities: I. Foundations and operationalization. CASE in Point, 2, 1-10. Retrieved August 25, 2011, from www.fippcase.org/caseinpoint/ caseinpoint_vol2_no2.pdf

Dunst, C. J., Bruder, M. B., Trivette, C. M., Hamby, D. W., Raab, M., \& McLean, M. (2001). Characteristics and consequences of everyday natural learning opportunities. Topics in Early Childhood Special Education, 21, 68-92. http://dx.doi.org/10.1177/027112140102100202

Filer, J. D., \& Mahoney, G. J. (1996). Collaboration between families and early intervention service providers. Infants \& Young Children, 9(2), 22-30. doi: 10.1097/00001163-199610000-00005

Florida Department of Education. (2017) Florida Early Learning and Developmental Standards. Retreived from: http://flbt5.floridaearlylearning.com/standards.html

Frey, B. (2018). The SAGE encyclopedia of educational research, measurement, and evaluation (Vols. 1-4). Thousand Oaks, CA: SAGE Publications, Inc. doi: 10.4135/9781506326139

Greenspan, S., \& Weider, S. (1997). Developmental Patterns and Outcomes in Infants and Children with Disorders in Relating and Communicating: A Chart Review of 200 Cases of Children with Autistic Spectrum Diagnoses. The Journal of Developmental and Learning Disorders, 7(1), 2-38.

Guralnick, M. J., \& Bruder, M. B. (2019). Early intervention. In Handbook of intellectual disabilities (pp. 717-741). New York: Springer.

Hanft, B. E., \& Pilkington, K. (2000). Therapy in natural environments: The means or end goal for early intervention? Infants and Young Children, 12,1-13. http://dx.doi.org/10.1097/00001163-200012040-00006

Howe, T.-H., \& Wang, T.-N. (2013). Systematic review of interventions used in or relevant to occupational therapy for children with feeding difficulties ages birth-5 years. American Journal of Occupational Therapy, 67(4), 405-412. https://doi.org/10.5014/ ajot.2013.004564
Imperatore, E., Chang, M.C., Gutiérrez, J., \& Gunter, J.S. (2016). Effectiveness of a sensory-enriched early intervention group program for children with developmental disabilities [Eficacia de un programa grupal de intervención temprana sensorialmente enriquecido para niños con discapacidades del desarrollo]. American Journal of Occupational Therapy, 70(5), 1-8. https://doi. org/10.5014/ajot.2016.018481

Kingsley, K., \& Mailloux, Z. (2013). Evidence for the effectiveness of different service delivery models in early intervention services. American Journal of Occupational Therapy, 67(4), 431-436. https:// doi.org/10.5014/ajot.2013.006171

Landa, R. J. (2018). Efficacy of early interventions for infants and young children with, and at risk for, autism spectrum disorders. International Review of Psychiatry, 30(1), 25-39. doi:http://dx.doi.org. ezproxylocal.library.nova.edu/10.1080/09540261.2018.1432574

Nock, M. K., Michel, B. D., \& Photos, V. I. (2007). Single-case research designs. In McKay, D. (Ed.), Handbook of research methods in abnormal and clinical psychology (pp. 337-350). New York, United States: Sage. Retreived from https://us.corwin.com/sites/default/ files/upm-binaries/19353_Chapter_22.pdf

Ottenbacher, K. J. (1984). Strategies for Evaluating Clinical Change: Implications for Practice and Research (clinical practice, occupational therapy, research design). American Journal ofOccupational Therapy 38(10):647-659. https://doi.org/10.5014/ajot.38.10.647

Parham, L. D., Roley, S. S., May-Benson, T. A., Koomar, J., Brett-Green, B., Burke, J. P., \& Schaaf, R. C. (2011). Development of a fidelity measure for research on the effectiveness of the Ayres Sensory Integration intervention. American Journal of Occupational Therapy, 65(2), 133-142. http://dx.doi.org/10.5014/ajot.2011.000745

Rosin, S. J., Saa, M. P. y Pavone, A. (2020). Un programa terapéutico interprofesional, basado en el juego, para grupos de niños con discapacidades del desarrollo. Revista Argentina de Terapia Ocupacional, 6(1), 20-28.

Salkind, N. J. (2010). Encyclopedia of research design. Thousand Oaks, CA: SAGE Publications, Inc. doi: 10.4135/9781412961288

Sandall, S., Hemmeter, M. L., Smith, B. J., \& McLean, M. (2005). DEC recommended practices: A comprehensive guide for practical application. Longmont, CO: Sopris West.

Tanner, T., Schmidt, E., Martin, K., \& Bassi, M. (2020) Interventions Within the Scope of Occupational Therapy Practice to Improve Motor Performance for Children Ages 0-5 Years: A Systematic Review. American Journal of Occupational Therapy, 74(2):7402180060. https://doi.org/10.5014/ajot.2020.039644

Workgroup on Principles and Practices in Natural Environments. (2008). Agreed upon practices for providing early intervention services in natural environments. OSEP TA Community of Practice: Part C Settings. Disponible en http://ectacenter.org/ pdfs/topics/ families/Finalmissionandprinciples3_11_08.pdf 
Zhan, S., \& Ottenbacher, K. J. (2001). Single Subject Research Designs for Disability Re-search. Disability and Rehabilitation, 23, 1-8. https://doi.org/10.1080/09638280150211202

Zwaigenbaum, L., Bauman, M. L., Choueiri, R., Kasari, C., Carter, A., Granpeesheh, D., et al. (2015). Early intervention for children with autism spectrum disorder under 3 years of age: Recommendations for practice and research. Pediatrics, 136, 60-81. 
